\title{
DEMON Acoustic Ship Signature Measurements in an Urban Harbor
}

\author{
Kil Woo Chung, Alexander Sutin, Alexander Sedunov, and Michael Bruno
}

Stevens Institute of Technology, Castle Point on Hudson, Hoboken, NJ 07030, USA

Correspondence should be addressed to Kil Woo Chung, kchung1@stevens.edu

Received 11 January 2011; Accepted 19 March 2011

Academic Editor: Abul Azad

Copyright () 2011 Kil Woo Chung et al. This is an open access article distributed under the Creative Commons Attribution License, which permits unrestricted use, distribution, and reproduction in any medium, provided the original work is properly cited.

Detection, classification, and tracking of small vessels are important tasks for improving port security and the security of coastal and offshore operations. Hydroacoustic sensors can be applied for the detection of noise generated by vessels, and this noise can be used for vessel detection, classification, and tracking. This paper presents recent improvements aimed at the measurement and separation of ship DEMON (Detection of Envelope Modulation on Noise) DEMON acoustic signatures in busy harbor conditions. Ship signature measurements were conducted in the Hudson River and NY Harbor. The DEMON spectra demonstrated much better temporal stability compared with the full ship spectra and were measured at distances up to $7 \mathrm{~km}$. The combination of cross-correlation and methods allowed separation of the acoustic signatures of ships in busy urban environments.

\section{Introduction}

Detection, classification, and tracking of small vessels are important tasks for improving port security and the security of coastal and offshore operations. DHS issued the Small Vessel Security Strategy [1] with one of the goals "to enhance maritime security and safety based on a coherent framework with a layered, innovative approach."

The research being conducted in the Center for Secure and Resilient Maritime Commerce (CSR), a DHS National Center of Excellence for Port Security, examines some basic science issues and emerging technologies to improve the security of ports as well as coastal and offshore operations. This research follows a layered approach utilizing above water and underwater surveillance techniques. The investigated layers include satellite-based wide area surveillance, HF Radar systems providing over-the-horizon monitoring, and nearshore and harbor passive acoustic surveillance. Integration of these systems is aimed at achieving vessel detection, classification, identification, and tracking. In recent field experiments in NY Harbor, the wide area sensing was provided by the University of Miami CSTARS facility's electrooptical (EO) and synthetic aperture radar (SAR) satellite imaging capabilities. The Stevens Passive Acoustic System was used for detecting sound produced by the same ships that were detected by satellites, and the advantages of the joint satellite/acoustics ship reconnaissance were examined [2]. The application of the passive acoustic method for small vessel detection, classification, and tracking in noisy and busy urban environments requires the development of novel methods of signal processing. These methods are presented in this paper.

Passive acoustic methods are based on the detection of sound produced by moving vessels. Passive acoustic methods have been applied for submarine detection for many years. There is a limited amount of publicly available publications covering the parameters of submarine sounds: many results were published just after World War II [3] and some acoustic parameters of Russian submarines are presented in [4]. Results of ship noise measurements can be found in many publications. A large amount of research has been conducted on the impact of the noise generated by ships on marine life and a good review of the work in this direction is given in [5]. A detailed description of the mechanisms of sound generation by ships can be found in $[6,7]$. According to [7], the main sources of vessel sound include the following:

(i) the prime movers-typically diesel engines, 


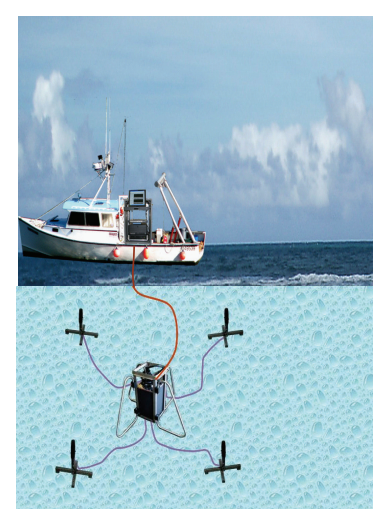

(a)

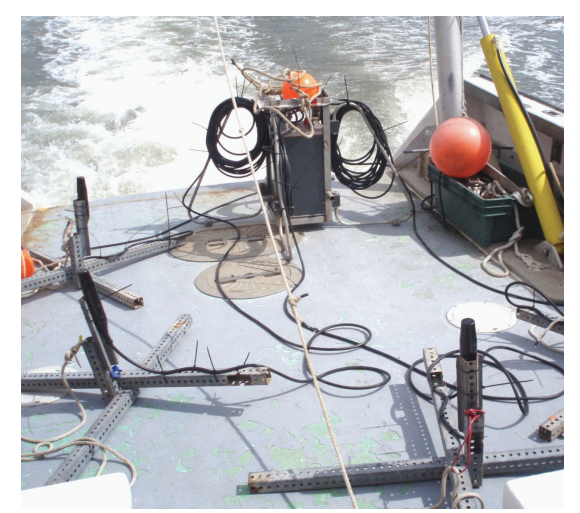

(b)

Figure 1: Schematic of the Stevens Passive Acoustic Detection System (a) and photo of the system on the deck of RV Savitsky prepared for deployment.

(ii) shaft-line dynamics,

(iii) propeller radiated pressures and bearing forces,

(iv) air conditioning systems,

(v) maneuvering devices such as transverse propulsion units,

(vi) cargo handling and mooring machinery,

(vii) vortex shedding mechanisms,

(viii) intakes and exhausts,

(ix) slamming phenomena,

Noise radiated by small vessels can be applied for small vessel detection, tracking, and classification. Various algorithms for these purposes were developed and some of them are presented in [8-10].

Among the various methods of ship noise detection and classifications one of the most reliable acoustic parameters is the spectrum of the ship noise envelope. The noise radiated by a ship is modulated at a rate dictated by some parameters of the propeller and engine (number of blades, rotational speed). Evaluation of that modulation provides information on the ship, such as the shaft rotation frequency, that can be used for ship classification. The method for estimation of the envelope modulation is known as DEMON (Detection of Envelope Modulation on Noise) [11-17] and the earlier papers describing this method were published about 50 years ago [11]. The DEMON spectra were the basis for various algorithms of ship classification.

However, the DEMON method in its current form cannot be used in cases where hydrophones receive signals from several ships simultaneously. We have addressed this shortcoming by developing a technique to separate the DEMON signatures using the cross-correlation of signals arriving at various hydrophones. The presented results are based on application of cross-correlation method for DEMON ship signature extraction $[18,19]$. These papers showed the applicability of the cross-correlation method for the separation of the ship acoustic signature in one simple example. The earlier suggested method was improved by using various time windows for ship tracking and acoustic signature extraction. The improved method was utilized for the measurement of DEMON signatures of various vessels in the Hudson River and NY Harbor. Various features of the DEMON spectra were investigated, including the temporal stability and the dependence on the carrier noise frequency.

The passive acoustic surface vessel surveillance tests presented in this paper were conducted by using the Stevens Passive Acoustic system initially developed for diver detection [20-22].

\section{Description of the Stevens Passive Acoustic System and Experiment}

2.1. The System Hardware and Software. The Stevens Passive Acoustic Detection System (SPADES) allows the passive acoustic detection, tracking, and classification of various surface and underwater sources of sound including surface vessels, swimmers, various types of divers, and unmanned underwater vehicles [20-22].

The SPADES is passive acoustic system providing the acquisition and analysis of sound generated by various sources. The system uses just four hydrophones and provides simultaneous acquisition and analysis of acoustical signals. The analysis function includes arbitrary digital filtering, spectral analysis, and cross-correlation for simultaneous processing of signals from several hydrophones, acoustical source separation, and determination of bearing for different targets relative to the central underwater mooring. The system also records and stores the complete raw acoustical data set, enabling further research and analysis of the acoustic signatures.

The system components include a land-based computer and an in-water system (Figure 1). The two systems are connected via an underwater cable that provides power and communication between the two systems.

The central mooring houses the electronic components required for signal conditioning, data acquisition, preprocessing, storage, and transmission. The four hydrophones are 


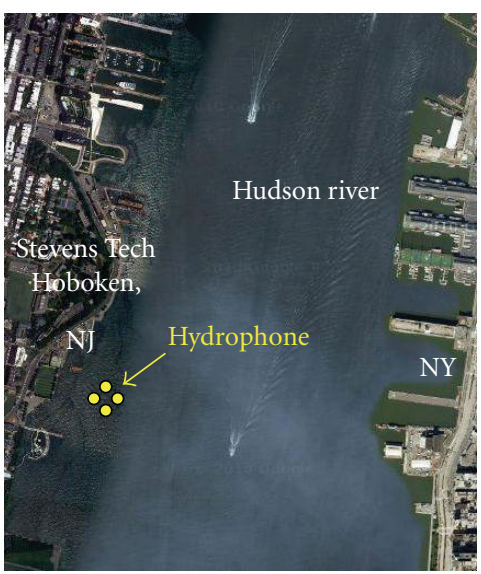

(a)

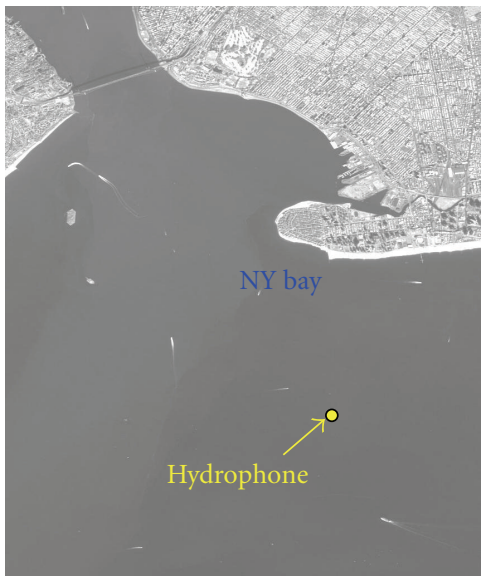

(c)

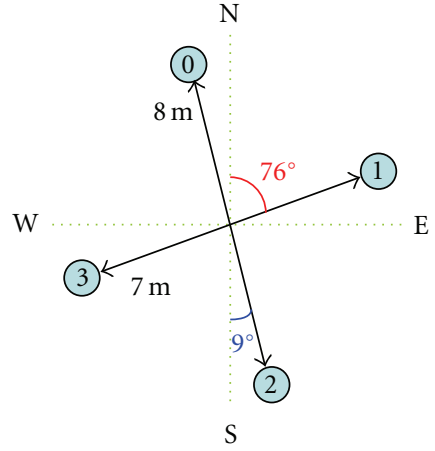

(b)

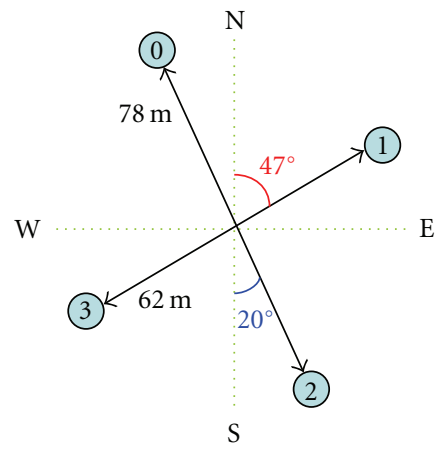

(d)

FIGURE 2: Map of the experiment sites and the SPADES hydrophone positioning.

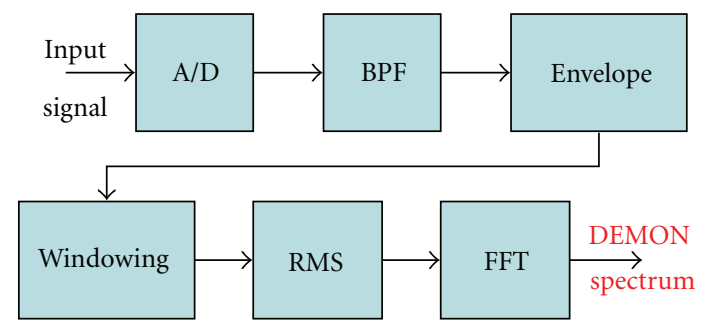

FIGURE 3: Block diagram of the DEMON method.

mounted on stands. Usually, the stands provide the hydrophone placement at a height of $60 \mathrm{~cm}$ above the bottom.

The system uses hydrophones manufactured by International Transducer Corporation-Model ITC-6050C. They are sensitive in the band of up to $100 \mathrm{kHz}$ and provide $-157 \mathrm{~dB}$ re $1 \mathrm{~V} / 1 \mu \mathrm{Pa}$ midband open circuit receiving response. The hydrophones are connected to the central mooring via underwater cables and can be deployed at distances up to $50 \mathrm{~m}$ from the central mooring.

The acoustic data from the hydrophones are acquired and recorded on the in-water system. There, it simultaneously undergoes preprocessing and is transmitted digitally to the land-based computer. The land-based computer further processes the data and displays the results in real time.

In this paper, we present results of experiments that were conducted in the Hudson River with the help of students from the Stevens Summer Research Institute (SRI) [23] in July 2010 and in a separate study conducted in lower NY Harbor in November 2009.

2.2. Experiments in the Hudson River and in NY Harbor. In our experiments we used four hydrophones in crossconfiguration. This configuration provides $360^{\circ}$ coverage of the investigated area. The planned distance between hydrophones was $10 \mathrm{~m}$ at the shallow water Hudson River test and $50 \mathrm{~m}$ for deeper case of the NY Harbor test. The hydrophones were dropped from the Stevens boat and real positions were slightly different from the planned positions.

The exact locations of the hydrophones were determined using a specially developed hydrophone positioning procedure. The procedure utilizes a supporting boat with GPS receiver, signal generator, and acoustical emitter. The vessel moves slowly and makes at least one full circle around 


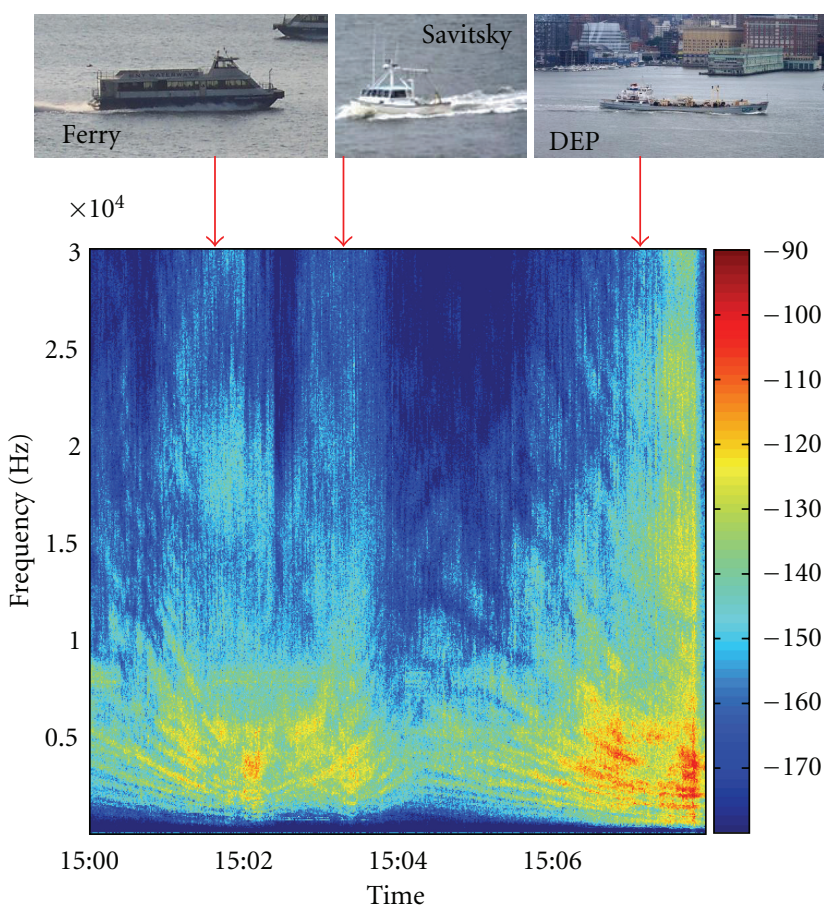

(a)

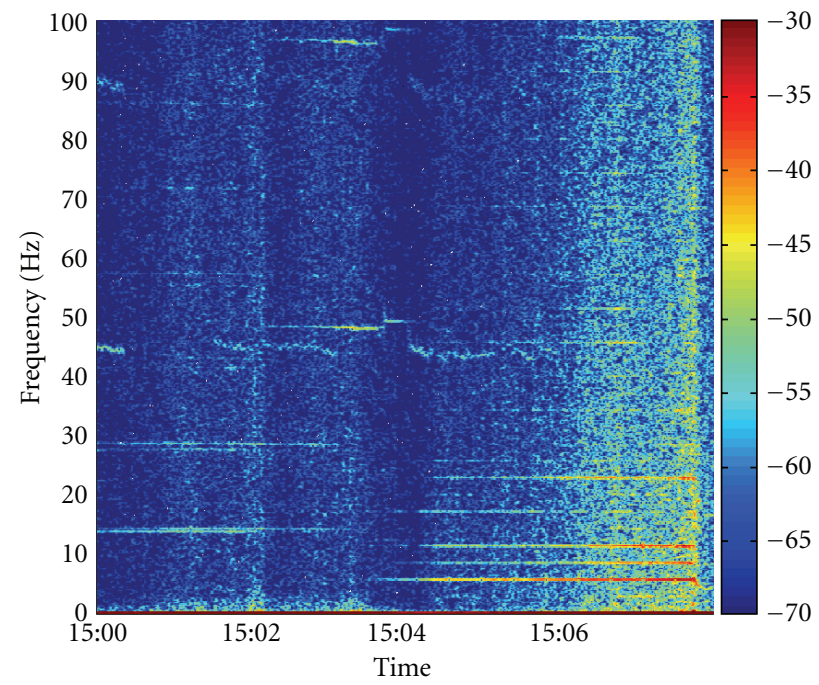

(b)

FIGURE 4: Full spectrogram (a) and DEMON spectrogram (b) for the time window from 15:00 to 15:10 (GMT) when three ships moved along the Hudson River. Photos of the ships are shown in the upper panel of the figure and arrows show time when the photos were taken.
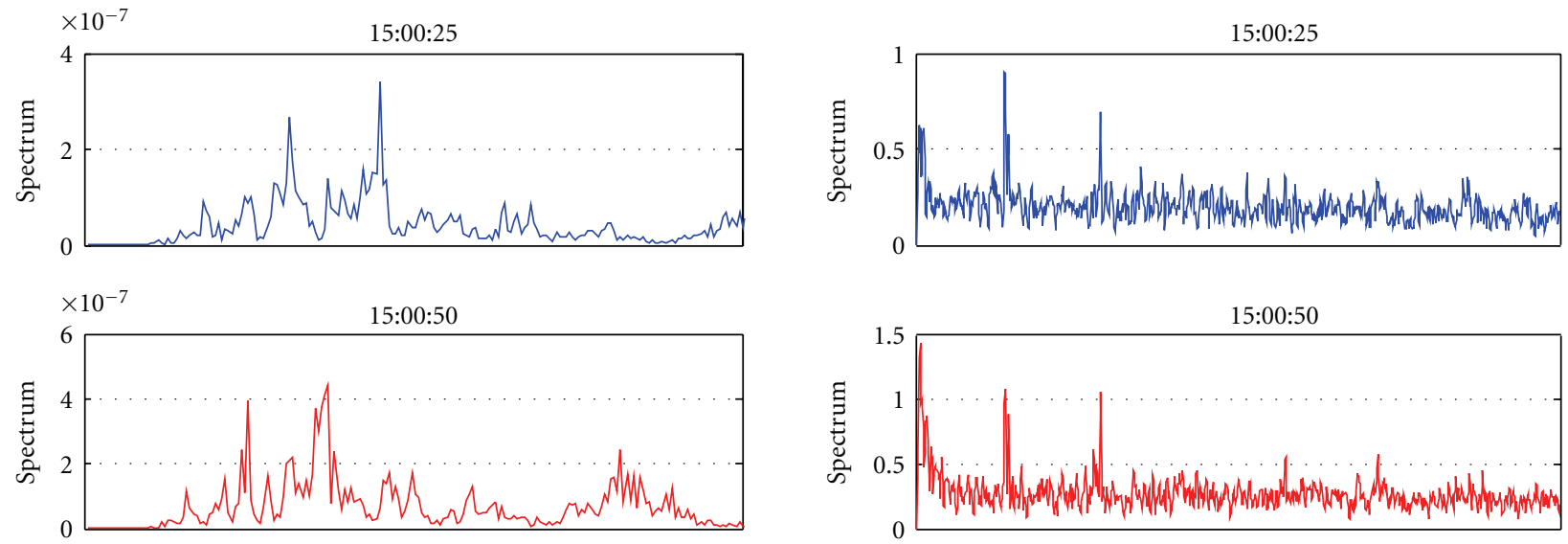

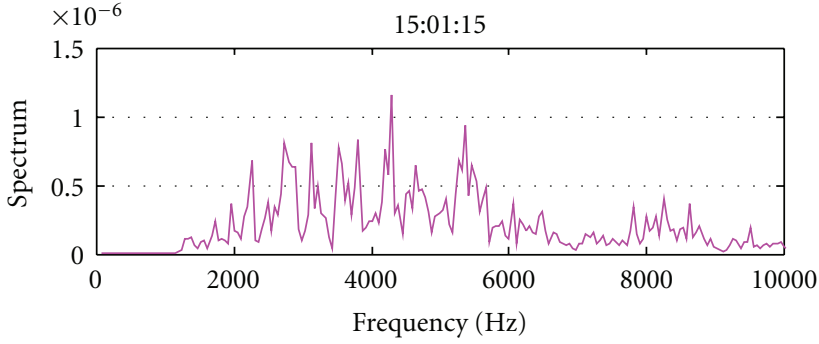

(a)

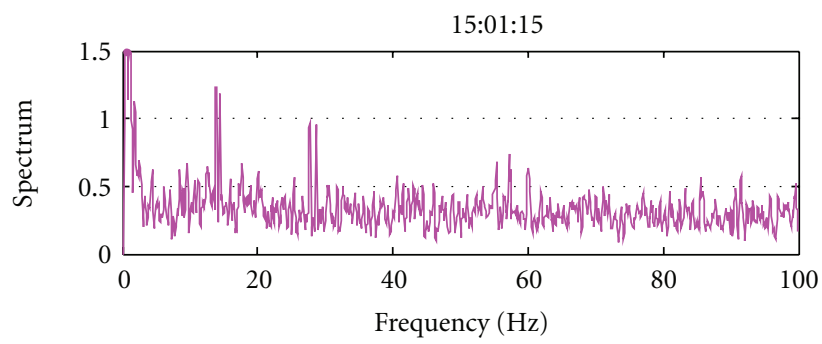

(b)

FIGURE 5: Comparison of the full spectrum (a) and the DEMON spectrum (b) in time for noise produced by a ferry. The shown spectra were recorded with 25 -sec and interval. 


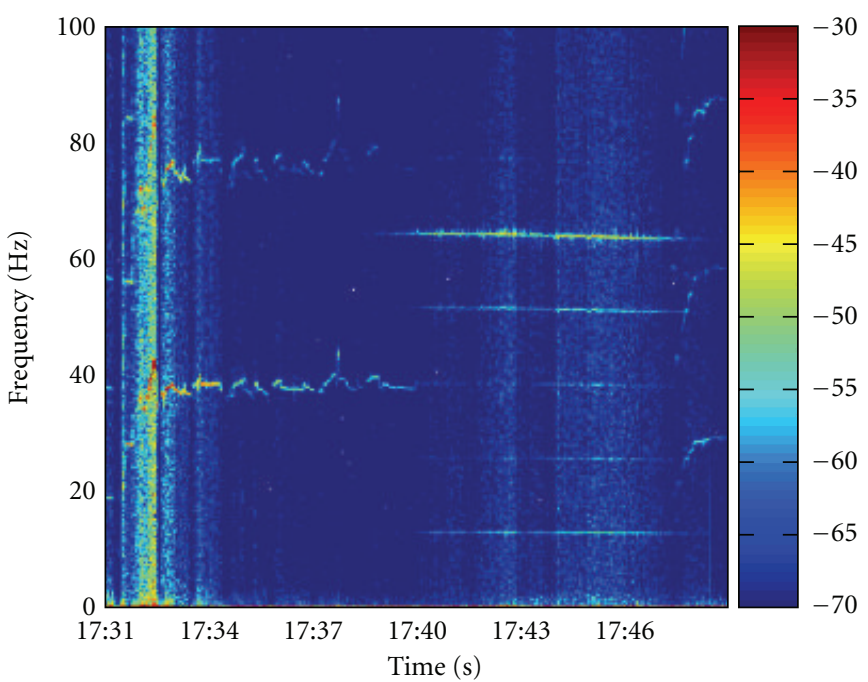

(a)

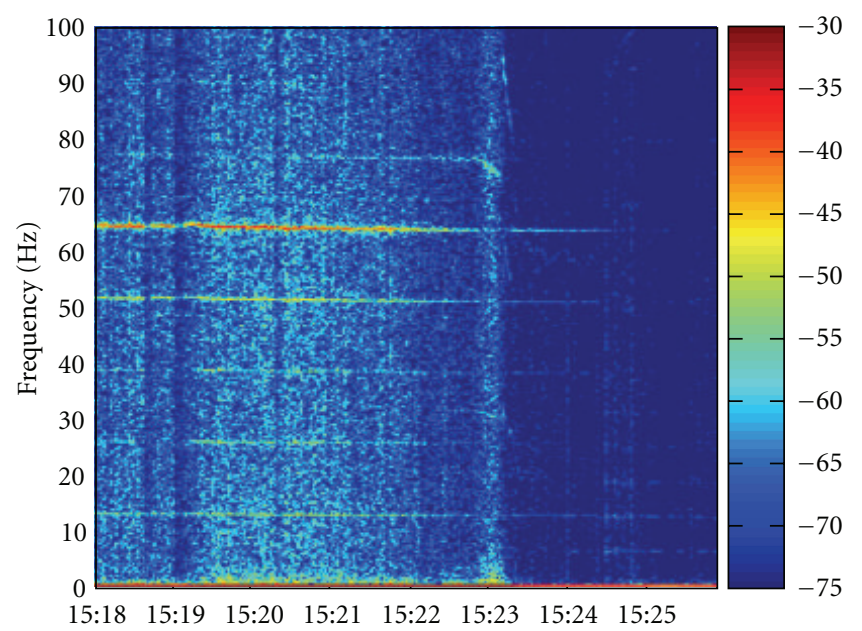

(c)

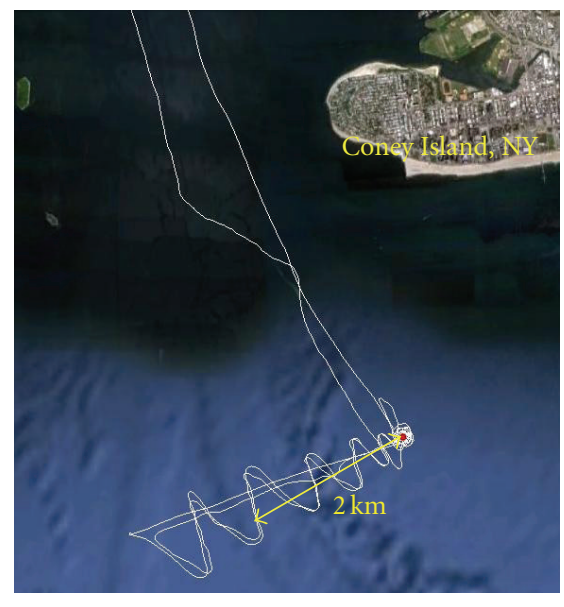

(b)

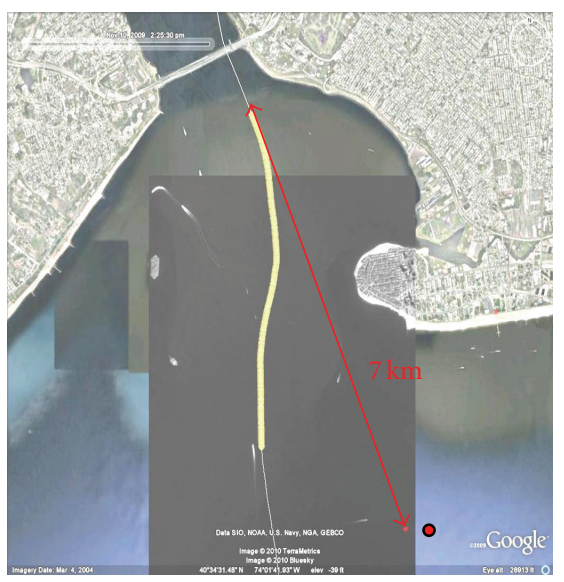

(d)

FIGURE 6: DEMON spectrogram and GPS tracking of the R/V Phoenix (a, b) and the SeaStreak (c, d).

the acoustic system, while emitting noise signals in the water and recording GPS coordinates along the way. The SPADES system software calculates the correlograms for various pairs of hydrophones and provides information about the time delay between acoustic signals arriving at the hydrophones. A specially developed program calculates the hydrophone positions that provide the least squares root deviation of the calculated time delays to the measured values. The accuracy of the hydrophone acoustic localization was about $1 \mathrm{~m}$.

In the Hudson River experiment, hydrophones \#0 and \#2 were oriented at $-9^{\circ}$ from the North-South axis and hydrophones \#1 and \#3 were oriented at $76^{\circ}$. The distance between hydrophones $\# 0$ and \#2 was $8 \mathrm{~m}$ and the distance between the other pair was $7 \mathrm{~m}$ (see Figures 2(a) and 2(b)).

In the NY Harbor experiment, the hydrophones were placed at Latitude 40.032.596' and Longitude -74.000.116'. The depth at the point of measurements was about $10 \mathrm{~m}$. Hydrophones $\# 0$ and $\# 2$ were oriented at $-20^{\circ}$ from the North-South axis and hydrophones \#1 and \#3 were oriented at $47^{\circ}$. The distance between hydrophones $\# 0$ and $\# 2$ was $78 \mathrm{~m}$ and the distance between the other pair was $62 \mathrm{~m}$ (see Figures 2(c) and 2(d)).

\section{Ship Noise and DEMON Acoustic Signatures}

Here, we present the results of DEMON signatures extraction and analysis for signals recorded by a single hydrophone.

\subsection{DEMON Algorithm Description. The applied DEMON} signal processing can be described in the following way.

The acoustic wave arriving at the hydrophone is transferred to the electrical analog signal $x(t)$. This signal is digitized with a sampling rate $f_{s}$; so the sampled signal is given by $x[i]=x(t=i T)$ where $i=0,1,2, \ldots$, and $T=1 / f_{s}$ is a sampling interval. In our experiments, the sampling rate was $200 \mathrm{kHz}$. The signal is bandpass filtered (BPF) in the frequency band $10-90 \mathrm{kHz}$. This filtering allows us to suppress high ambient noise in the lowfrequency band. 


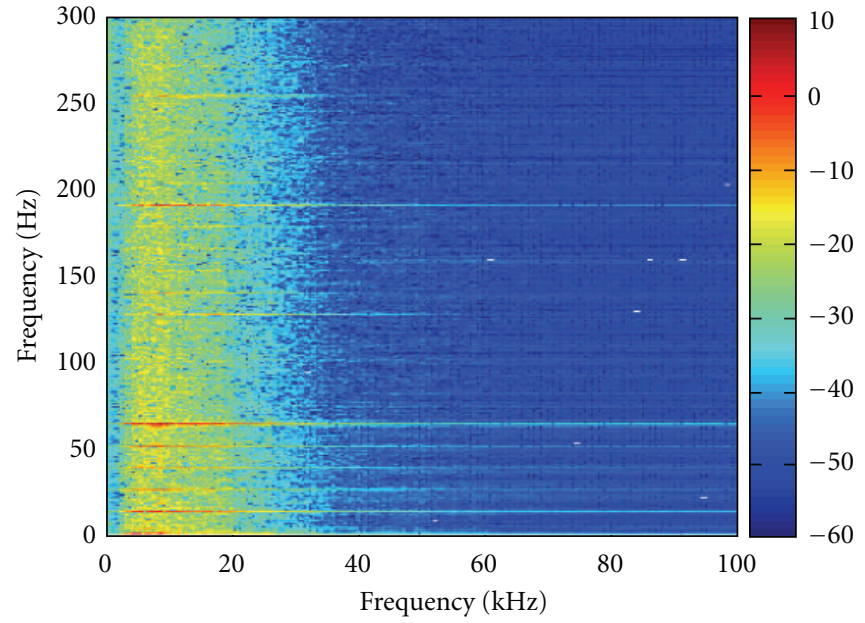

(a)

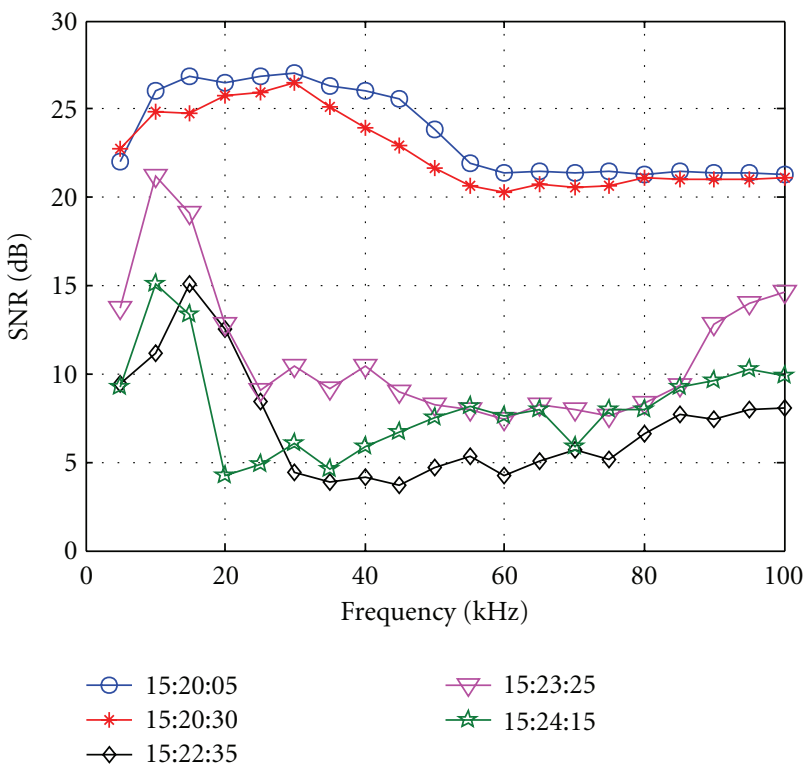

(c)

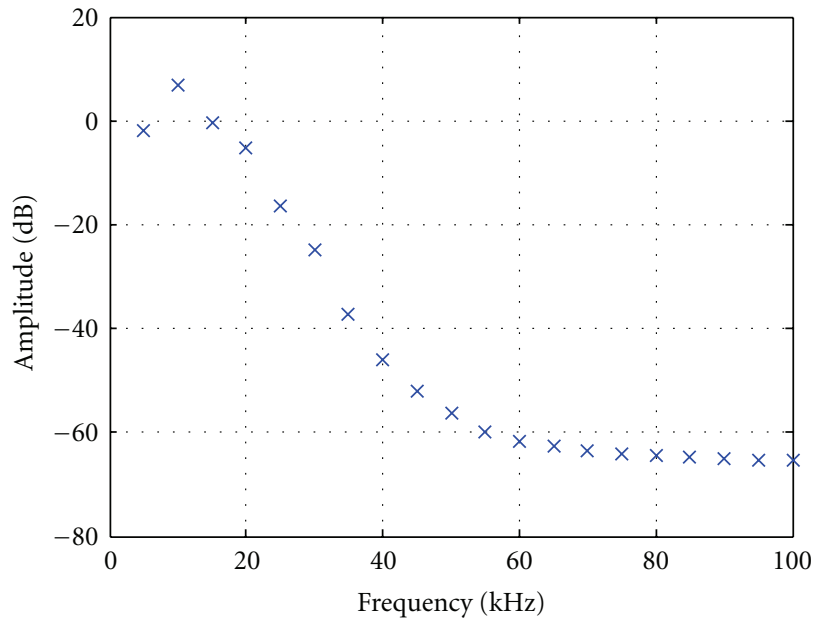

(b)

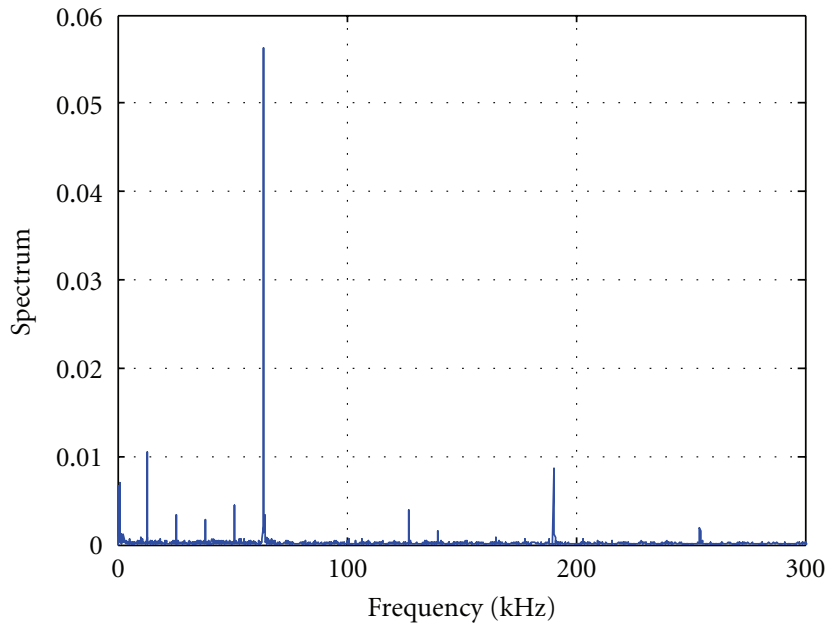

(d)

Figure 7: (a) The dependence of the DEMON spectra (DEMON frequency is the vertical axis, spectral amplitude shown by color) from the carrier frequency (the horizontal axis), (b) dependence of the amplitude of DEMON frequency component $63.6 \mathrm{~Hz}$ on the carrier frequency, (c) SNR dependence on frequency at various measurement distances, and (d) DEMON spectrum at optimized frequency band (25-30 kHz) at short distance.

The real envelope of the digitized filtered signal $\hat{x}[i]$ is calculated using a Hilbert transform and the average value of the envelope is computed using the root mean square (RMS) in the time window $T_{D}$. This time window contains $N=T_{D} f_{s}$ samples and the averaged envelope is calculated by the following formula:

$$
z[l]=\sqrt{\frac{1}{N} \sum_{i=l N}^{(l+1) N-1} \hat{x}^{2}[i] .}
$$

We computed the averaged signal envelope with a sampling rate $f_{D}=1 / T_{D}$. In the results presented below, the DEMON sampling rate was $2 \mathrm{kHz}$.
The FFT of the calculated envelope presents the DEMON spectrum that we are interested in. The block diagram of this method is shown in Figure 3. The spectral properties of the envelope signal can be presented in the form of spectrograms and spectra.

3.2. Temporal Stability of the DEMON Signatures. Figure 4 presents results of the spectral analysis of acoustic signals in the Hudson River recorded on July 3, 2010 from 15:00 to 15:10 (GMT). During this time, three ships (a Fast Ferry, the Stevens research vessel Savitsky, and an NY DEP barge) moved along the river.

The full spectrogram (Figure 4(a)) does not contain horizontal lines that are indicators of narrow frequency spectral 


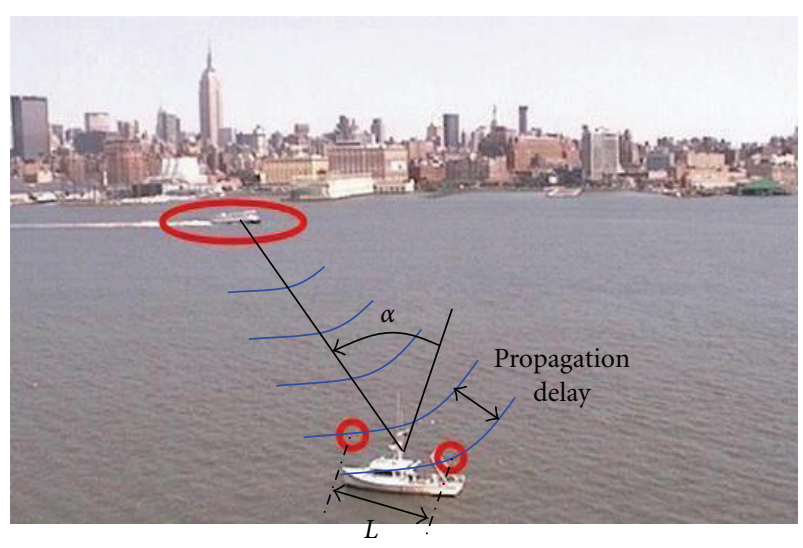

FIGURE 8: The noise radiated by a source reaches the hydrophones separated by distance $L$ with a delay that depends on the direction of the source with respect to the line perpendicular to the hydrophone axis.

component. At the same time the DEMON spectrogram (Figure 4(b)) has several narrow frequency components indicated as horizontal lines. These narrow frequency components can be used for ship classification.

Comparison of the full spectrum and the DEMON spectrum recorded at slightly different time windows is presented in Figure 5. At this time, the acoustic noise was produced by a passing ferry. It is seen that the full spectrum of the ship noise has large temporal variation while the spectral components of the DEMON spectrum are practically the same. The presented examples demonstrate that the DEMON spectra are much more reliable for ship identification than the full acoustic spectra.

3.3. Detection Distance of the DEMON Signatures. Here we present two examples showing the estimation of the maximal distances for observation of the DEMON ship acoustic signatures. The presented examples show the signals recorded in lower NY Harbor, where the sound attenuation is less than in the Hudson River.

Figures 6(a) and 6(b) shows the DEMON spectrogram recorded on November 9, 2009 from 17:37:00 to 17:46:00 (GMT). During this time, the Stevens research vessel Phoenix moved in a zigzag pattern away from the hydrophone system. The DEMON spectrum of Phoenix was detected until 17:40:00 and the detection distance of the Phoenix was about $2 \mathrm{~km}$.

The DEMON spectrogram and GPS tracking of the SeaStreak recorded on November 9, 2009 from 15:18:00 to 15:26:00 (GMT) are shown in Figures 6(a) and 6(b). The DEMON signature was detected until 15:25:00 and the SeaStreak was at distance of approximately $7 \mathrm{~km}$ from the hydrophone system at that time.

Larger detection distance for SeaStreack in comparison with the Phoenix is connected with higher noise generated by SeaStreak. Phoenix is several times smaller and had speed about twice less than SeaStreak.
3.4. Optimization of Frequency Band for the DEMON. The presented DEMON signal processing was conducted for the acoustic signals filtered in the band $10-90 \mathrm{kHz}$. Choosing the optimal frequency band can improve the Signal/Noise Ratio (SNR) and increase the detection distance. We calculated the dependence of the DEMON spectra on the carrier noise frequency using sequential bandpass filtering with $1 \mathrm{kHz}$ band in the DEMON algorithm presented in Figure 3. The results of this calculation for the acoustic noise produced by the SeaStreak ferry are presented in Figure 7(a). This figure shows the dependence of the DEMON spectra (DEMON frequency is the vertical axis, and spectral amplitude is shown by color) on the carrier frequency (the horizontal axis). It is seen that the DEMON component amplitudes decrease with the carrier frequency. The dependence of the single frequency component $(63.6 \mathrm{~Hz})$ on the carrier frequency is presented in Figure 7(b).

Just as the amplitudes of the DEMON frequency components are decreased with the frequency, the noise level is also decreased. Therefore, for funding the optimal frequency providing the maximal SNR, we estimated the frequency dependence of the SNR. The SNR was estimated as a ratio of the amplitude of the single frequency component $(63.6 \mathrm{~Hz})$ to the average level of the other frequency components (70$170 \mathrm{~Hz}$ ) in the DEMON spectra. We computed the average SNR for every $5 \mathrm{kHz}$ frequency band of the ship noise carrier signal. The calculated SNR is shown in Figure 7(c) for various times when the SeaStreak moved away from the hydrophone system. It is seen that the maximal value of SNR was in the range $20-40 \mathrm{kHz}$ at short distances to the boat and decreased to the band $10-20 \mathrm{kHz}$ for larger distances. The decreasing of optimal frequency with the distance can be explained by lower acoustic attenuation of lower frequencies. Figure 7(d) presents an example of the DEMON spectrum at optimized frequency band.

\section{Cross-Correlation Method of Ship Tracking and DEMON Signature Extraction}

The SPADES system developed at Stevens utilizes signals coming from four hydrophones. Even using a small number of hydrophones, the acoustic source detection and bearing determination can be accomplished by the calculation of the cross-correlation of acoustic signals recorded by various pairs of hydrophones. This section of the paper considers how the measurements of cross-correlation can be used for the estimation of bearing to a source of sound and how the crosscorrelation method allows the separation of the DEMON signatures in busy environments.

4.1. Cross-Correlation for Ship Tracking. Let us consider the signals received by two hydrophones separated by a distance $L$. They record the noise radiated by a source whose direction makes an angle with the normal to the line between the hydrophones. The distance between the source and the hydrophones is much larger than $L$. An example of such a configuration is illustrated in Figure 8. The noise radiated 


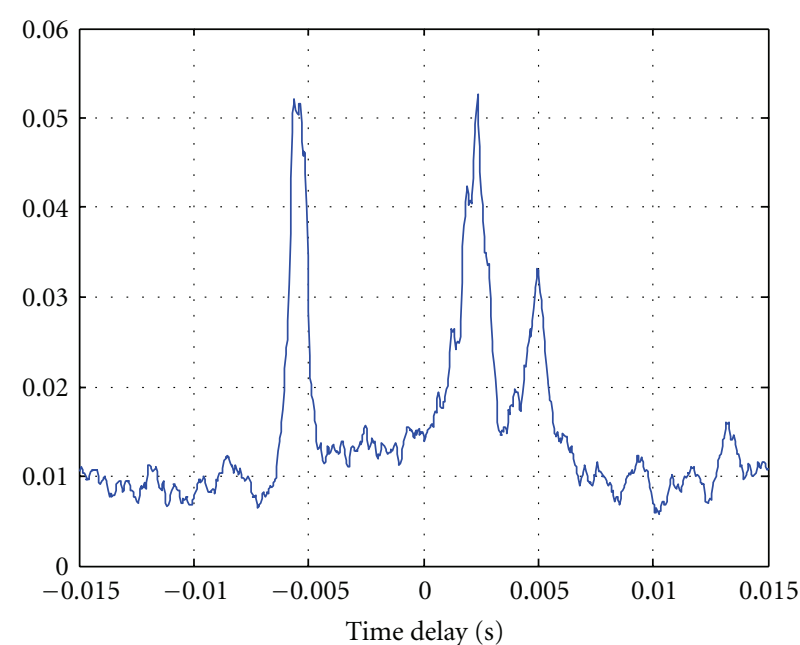

(a)

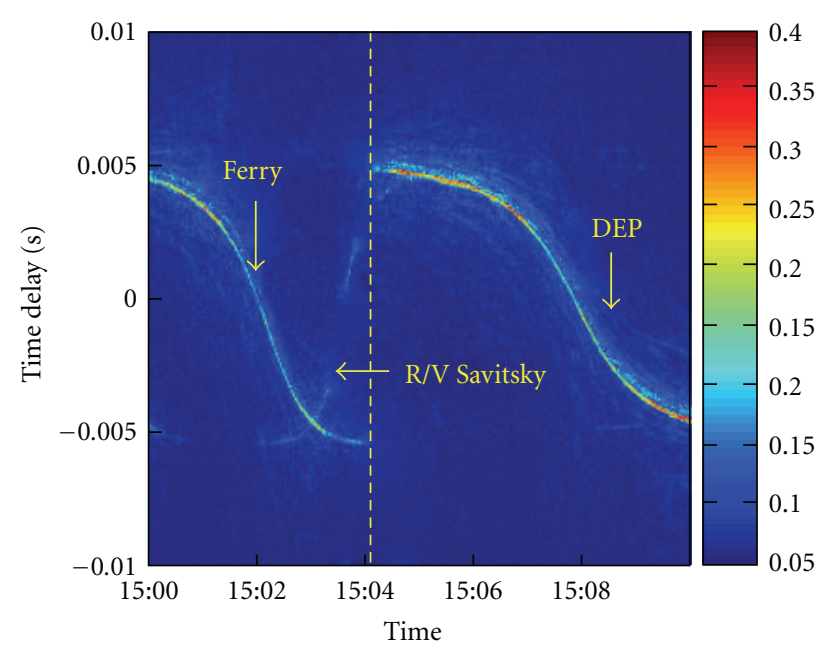

(b)

Figure 9: Example of the cross-correlogram for the same time window when the spectrograms presented in Figure 4 were taken (b) and cross-correlation of the received signal (a) for time shown by dotted yellow line in (b).

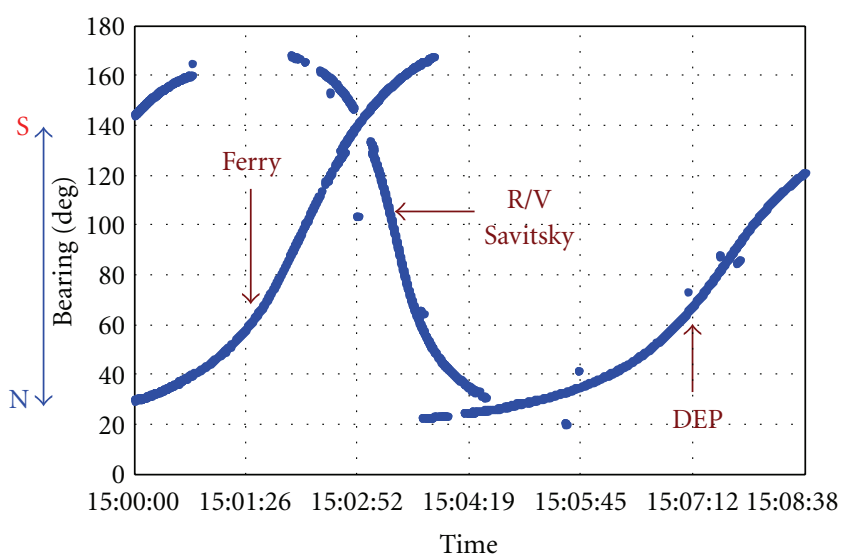

FIGURE 10: Acoustic ship tracking using cross-correlation.

from the ship reaches the two hydrophones with a delay $\Delta T$ between them:

$$
\Delta T=\frac{L \sin \alpha}{c},
$$

where $c$ is the speed of sound in water.

Let us assume that a single vessel contributes to the acoustic field and that the signals $x(t)$ and $y(t)$ recorded by the two hydrophones are delayed versions of the same signal:

$$
y(t)=x(t-\Delta T),
$$

and $\Delta T$ is the delay introduced in (3). The cross-correlation of the signals is defined as

$$
R(\tau)=\int_{-\infty}^{\infty} x\left(t^{\prime}\right) y\left(\tau-t^{\prime}\right) d t^{\prime} .
$$

For two delayed signals of the form (3),

$$
R(\tau)=A(\tau-\Delta T),
$$

where $A(\tau)=\int_{-\infty}^{\infty} x\left(t^{\prime}\right) x\left(\tau-t^{\prime}\right) d t^{\prime}$ is the autocorrelation function of $x(t)$. The cross-correlation $R(\tau)$ of the signals from the two hydrophones is the same as the autocorrelation $A(\tau)$ of the signal from hydrophone 1, but shifted to the time $\tau=\Delta T$. Because the autocorrelation of a signal is maximum at $\tau=0$, the cross-correlation is maximum at $\tau=\Delta T$. This means that the time location of the maximum of the crosscorrelation can be used to estimate the direction to the ship.

The time delays in the cross-correlation function can be recalculated to the source bearing angle using (2):

$$
\begin{gathered}
\alpha_{1}=\arcsin \left(\frac{c \Delta T}{L}\right), \\
\alpha_{2}=\pi-\arcsin \left(\frac{c \Delta T}{L}\right) .
\end{gathered}
$$

It is seen that the same time delay takes place for two possible angles, which produces ambiguities in the processing of the source bearing measurements by a single pair of hydrophones. This ambiguity can be solved using signal processing of several cross-correlation signals.

For ship tracking, we present the crosscorrelogram in the form of a floated chart similar to a spectrogram, a graph with two geometric dimensions: the horizontal axis represents time; the vertical axis is the delay between two hydrophone signals; a third dimension indicates the amplitude of the cross-correlation function. Figure 9 presents an example of the cross-correlogram for signals recorded in the Hudson River for the sensors \#0 and \#2. The cross-correlogram is shown for the same time window when the spectrograms presented in Figure 4 were taken.

The time delays of the detected peaks were recalculated to the ship bearing according to (6). To eliminate the ambiguity of the bearing for any hydrophone pair we used joint signal processing for several pairs. For our system having four hydrophones, the six various pairs can be combined. Any pair gives two values for the bearing-the real one and 


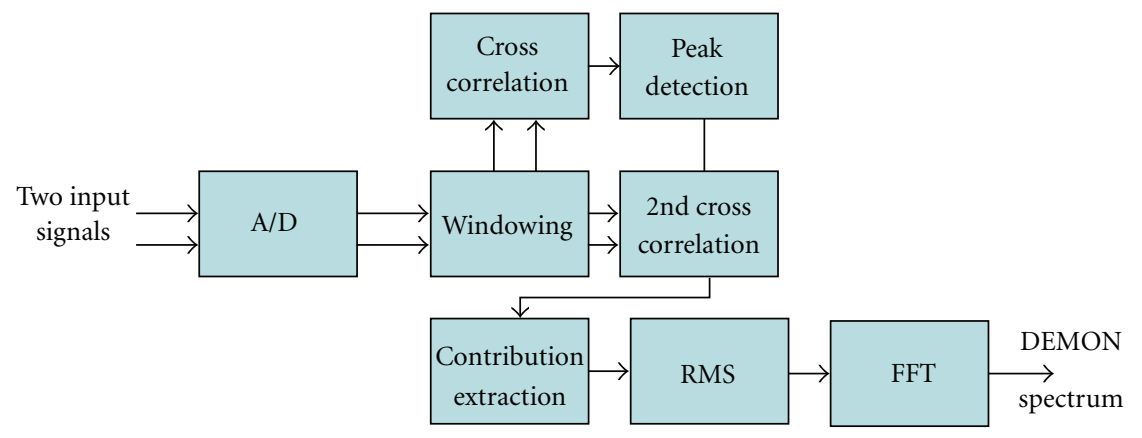

FIGURE 11: Block diagram of the cross-correlation DEMON method.

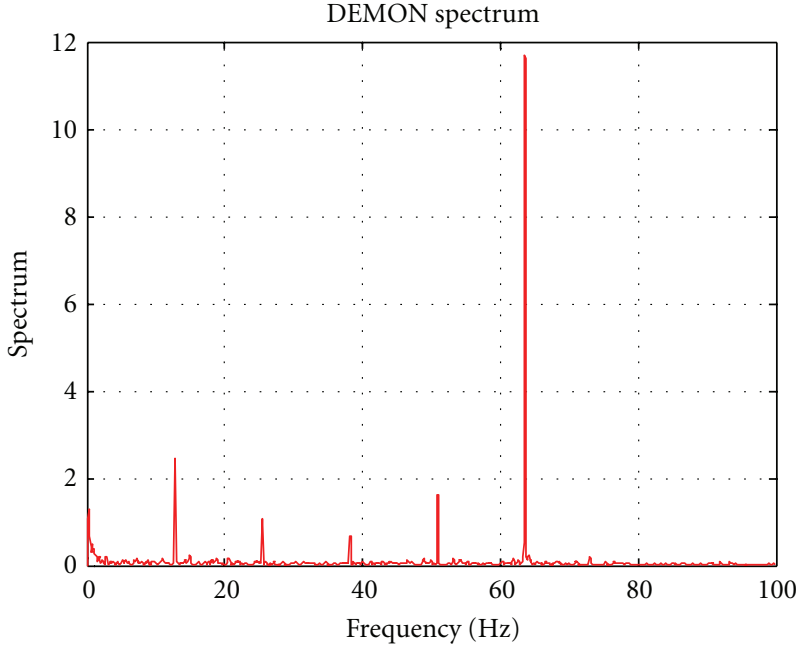

(a)

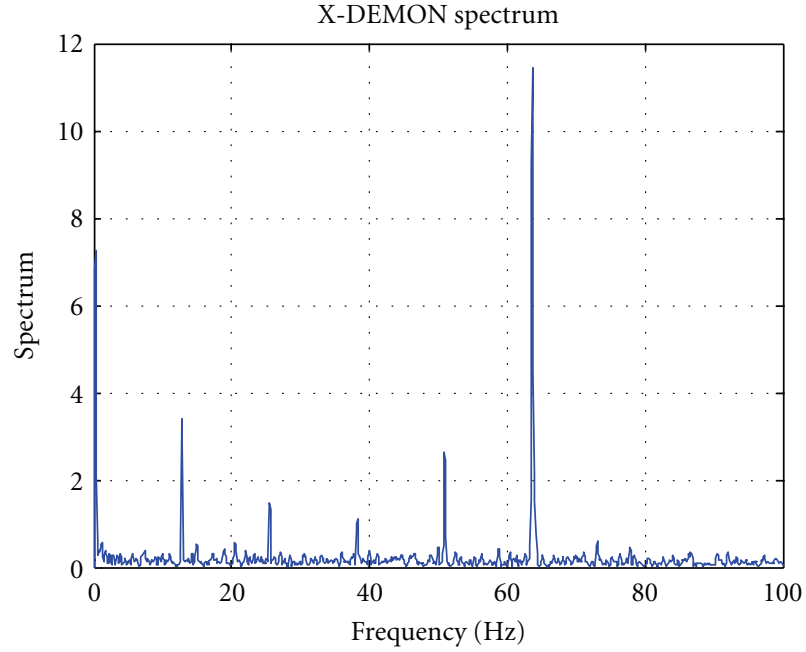

(b)

FIGURE 12: Comparison of the standard DEMON spectrum (a) and the cross-correlation DEMON spectrum (b) computed for the SeaStreak signal.

the imaginary one. The real bearings are the same for various hydrophone pairs while the imaginary bearings are different. This phenomenon was used for the separation of real and imaginary bearings. The multipair fusion algorithm also uses the fact that accuracy of the bearing determination is different for different pairs. As is seen from (6) the dependence of angle on delay is nonlinear and as such the accuracy of the angle determined by one cross-correlation pair varies with angle, as can be seen by computing the rate of angle change per unit delay:

$$
\frac{d \alpha}{d \tau}=\frac{d \arcsin (c \tau / L)}{d \tau}=\frac{c}{L \sqrt{1-(c \tau / L)^{2}}} .
$$

It is seen that small errors in the delay $\tau$ lead to smaller errors in the bearing finding for angles close to the normal of the line connecting the two sensors $(a \rightarrow 0, \tau \rightarrow 0)$. The accuracy of the bearing finding decreases with the angle increasing and becomes very low for angles close to $90^{\circ}(\tau \rightarrow$ $L / c)$. In the developed software, the bearing determination is conducted using hydrophone pairs that provide the highest accuracy for any target. This means that the bearing to any target is determined by the hydrophone pair having a higher value of $\alpha^{\prime}(\tau)$ for the angle at which the target is detected.

The hydrophone pair with higher value of $\alpha^{\prime}(\tau)$ determines two possible bearings_-one of them is real and the second is imaginary. As noted earlier, in order to isolate the real one we use information from other hydrophone pairs. The determined angles are recalculated back into the corresponding time delays predicted for a signal arriving from that angle via (3) for all hydrophone pairs. These recalculated time delays are compared with measured time delays. For real directions toward the targets, the measured time delays are clustered around the recalculated time delays, while for imaginary targets, the recalculated and measured time delays do not produce clusters and thus can be discarded.

Figure 10 presents an example of the vessel tracks determined using the developed software. These tracks were received at the time window from 15:00 to 15:10 (GMT) on July 3,2010 . Photos of the vessels and their acoustic signatures are show in Figure 3. 


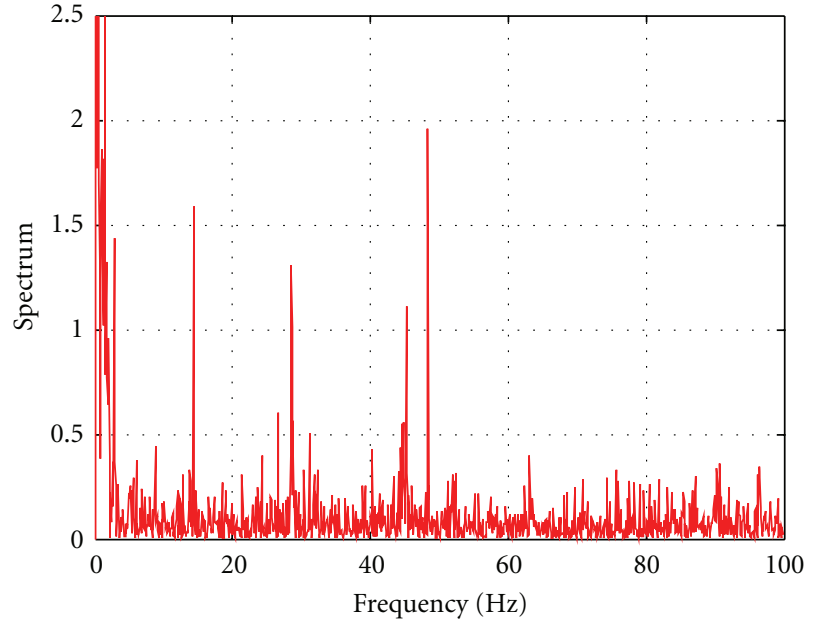

(a)

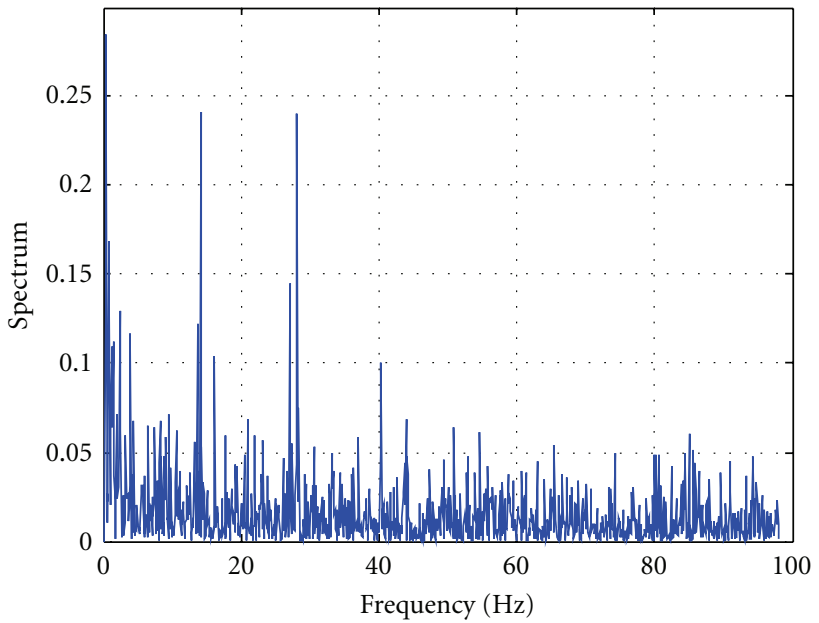

(b)

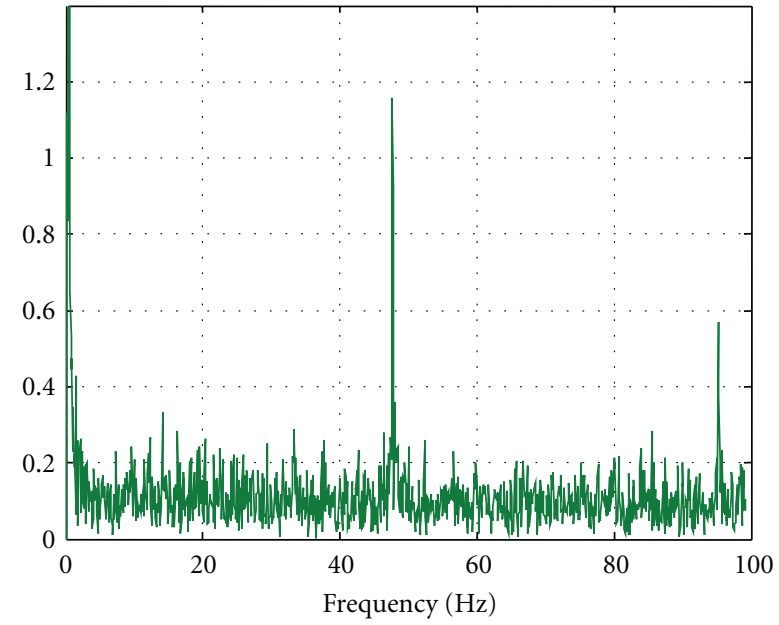

(c)

Figure 13: Example of separation of ferry and Savitsky spectra from the mixed signals: (a) the DEMON spectrum of the mixed signals (ferry + Savitsky), (b) the separated ferry X-DEMON spectrum, and (c) the separated Savitsky X-DEMON spectrum.

4.2. DEMON Spectrum Finding Using the Cross-Correlation. The application of the cross-correlation allows extraction of the acoustic signal from definite vessels in the presence of many acoustic targets. It can be done for acoustic targets having different bearings.

Stevens has developed the cross-correlation method for DEMON ship signature extraction $[18,19]$. This method is based on the measurements of the cross-correlation function with relatively fast repetitions. The energy of the crosscorrelation signal around any peak is proportional to the energy of the noise radiated from the definite direction. Time variation of this signal presents the envelope of noise energy and the spectrum of the energy around the crosscorrelation peak is the same as the DEMON spectrum. We call this the X-DEMON spectrum. The developed algorithm calculates the envelope of the cross-correlation function in a relatively short time window ( 5 milliseconds). This short time window does not provide enough accuracy for ship tracking, but longer windows did not allow extraction of DEMON with the required sampling rate $(200 \mathrm{~Hz})$.

There is a contradiction in the choosing of the crosscorrelation time window. The relatively long time window (20 milliseconds and longer) is required for improving SNR and small errors in the bearing funding. This long time window does not provide measurements rate enough for DEMON signature measurements that has to be at least $200 \mathrm{~Hz}$. The sampling rate of $200 \mathrm{~Hz}$ can be reached using 5 milliseconds time window with $50 \%$ of overlapping.

To solve this problem, we applied different time windows for ship tracking and for DEMON signature extraction.

The cross-correlation calculation was conducted with two different sliding time windows (one is the long time window and the other is the short time window). The long time window was used for determining the accurate time delay using the cross-correlation. This time delay was applied to the other cross-correlations in the short time window to extract the DEMON signature. 


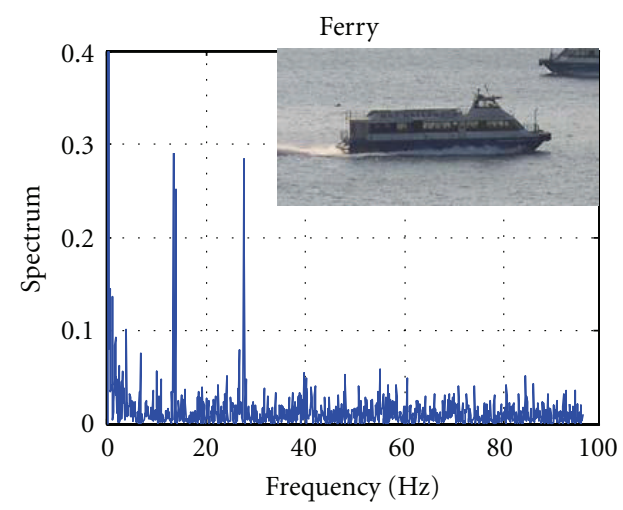

(a)

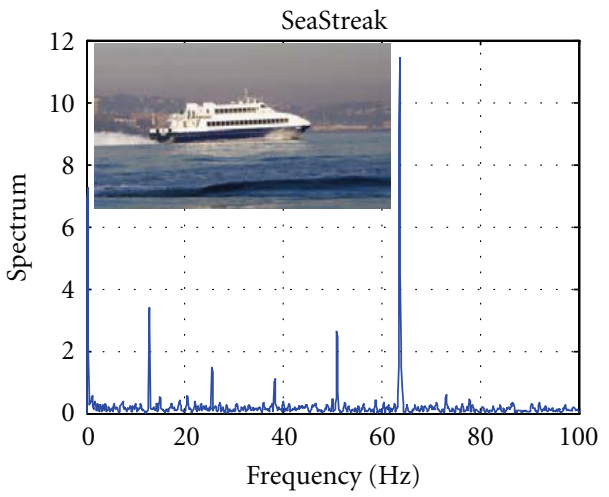

(c)

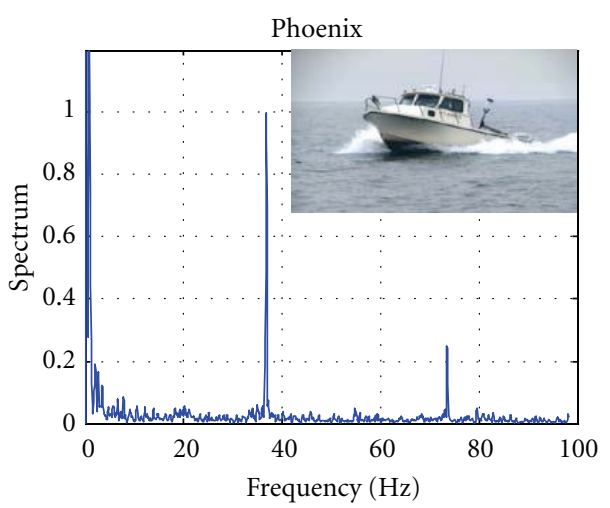

(e)

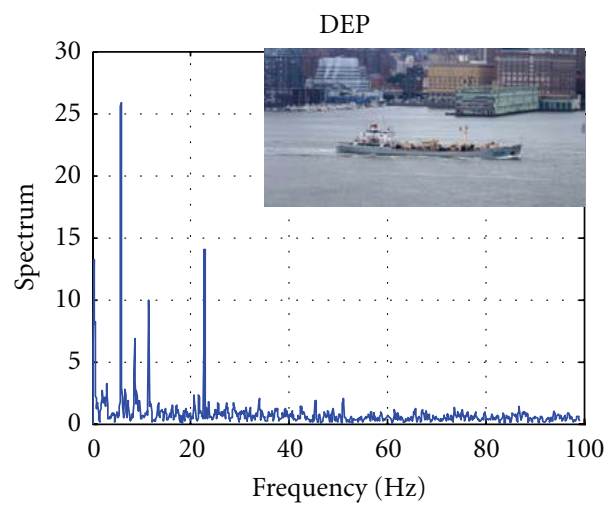

(b)

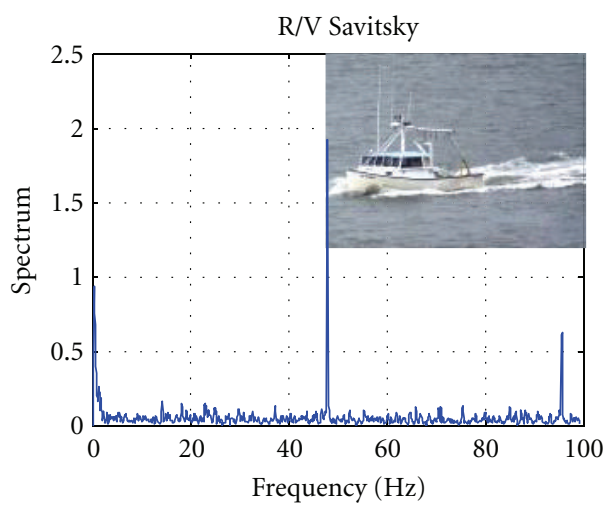

(d)

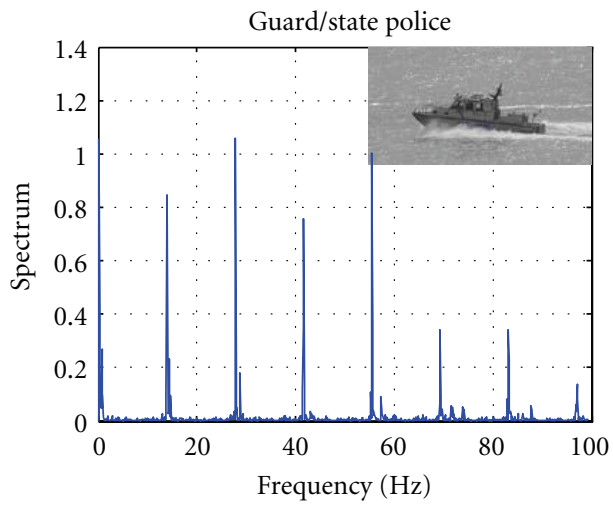

(f)

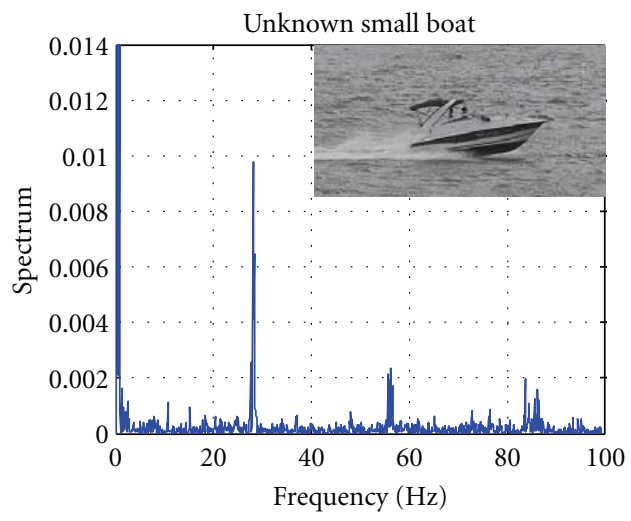

(g)

FIGURE 14: DEMON spectra of passing boats. 
We now describe how to compute the X-DEMON spectrum in detail. First, for discrete time signal processing, the continuous signals $x(t)$ and $y(t)$ were sampled with a sampling rate $f_{s}(=1 / T)$. The sampled signals were given by $x[i]$ and $y[i], i=0,1,2, \ldots$. These sampled signals were divided by using two different time windows with the same repetition time $T_{W}$. The X-DEMON sampling rate is therefore $1 / T_{W}$. Suppose that the long time window had $N$ samples, and the short time window had $P$ samples $(N>P)$. We used $50 \%$ overlapping for calculation of DEMON using the short time window. This means that $P=2 T_{W} f_{s}$. Let the signal within each sliding window be referred to as a frame. The crosscorrelation function of the $l$ th frame in the long time window can be denoted by

$$
R_{l}[m]=\frac{1}{N} \sum_{i=l P / 2}^{l P / 2+N-1} x[i] y[m-i], \quad l=0,1,2 \ldots
$$

From (8), we extracted the peak index $m_{l}$, having a local maximum value, which can be determined by

$$
m_{l}=\operatorname{argmax}\left(R_{l}[m]\right)
$$

We used the index $m_{l}$ to extract the contribution part in the second cross-correlation with short time window. Before the cross-correlation, the sampled $y[i]$ is shifted by $m_{l}$. The second cross-correlation is thus given by

$$
\hat{R}_{l}\left[m-m_{l}\right]=\frac{1}{P} \sum_{i=l P / 2}^{l P / 2+P-1} x[i] y\left[m-m_{l}-i\right] .
$$

From (10), the cross-correlation has a peak at $m=0$. And then, we compute the average value using the root mean square (RMS) over an interval $-M \leq m \leq M$ as

$$
\hat{z}[l]=\sqrt{\frac{1}{2 M+1} \sum_{m=-M}^{M}\left(\hat{R}_{l}\left[m-m_{l}\right]\right)^{2}}
$$

Calculation of the RMS of the cross-correlation function gave vectors presenting the envelope of the DEMON ship signature. The interval $|M|$ is small enough for extraction of the contribution in order to remove other noise signals which can decrease the efficiency of DEMON. We therefore use $50-100(0.1-0.5 \mathrm{~ms})$ sequences.

The FFT of the calculated envelope presents the DEMON spectrum that we are interested in. The block diagram of the cross-correlation DEMON method is shown in Figure 11. Figure 12 shows an example of DEMON spectrum and XDEMON spectrum computed for noise generated by the SeaStreak ferry. It is seen that the X-DEMON spectrum is very similar to the DEMON spectrum.

In our signal processing, the long time window was $200 \mathrm{~ms}$, the short time window was $10 \mathrm{~ms}$, and the time interval $T_{W}$ between the adjacent windows was $5 \mathrm{~ms}$. The envelop DEMON spectra were calculated for 10 second time window.
In order to demonstrate the efficiency of ship signature separation using the cross-correlation method, we used acoustic signals coming from the ferry and Savitsky at the same time (see Figure 4). An example of DEMON separation of the mixed signals recorded in the Hudson River in July, 2010 from 15:02:20 to 15:02:30 is presented in Figure 13. Figure 13(a) shows the standard DEMON spectrum of the mixed signals and Figures 13(b) and 13(c) are the separated $\mathrm{X}$-DEMON spectra of the ferry and Savitsky using the crosscorrelation approach.

Figure 13(a) shows narrow spectral component of the both ships together. The separation of these components for two ships using X-DEMON method is shown in Figures 13(b) and 13(c).

The examples of X-DEMON spectra of passing vessels are presented in Figure 14 for signals recorded in the Hudson River on July 3, 2010 and in NY Harbor on November 9, 2009. The calculated DEMON spectra show very different patterns for these different types of vessels.

\section{Conclusion}

We have demonstrated the effectiveness of the DEMON method for vessel detection and classification in busy environments. The DEMON method improves the detection distance and provides information for ship classification. The developed DEMON cross-correlation algorithm has been shown to be an efficient technique for the extraction of the ship acoustic signatures in the presence of several targets. The planned next step is the collection of a library of vessel acoustic signatures and the development of an automated vessel classification system.

\section{Acknowledgment}

This work was funded by the U.S. Department of Homeland Security under Grant Award no. 2008-ST-061-ML0002. The views and conclusions contained in this paper are those of the authors and should not be interpreted as necessarily representing the official policies, either expressed or implied of the U.S. Department of Homeland Security.

\section{References}

[1] Department of Homeland Security, "Small Vessel Security Strategy," April 2008, http://www.dhs.gov/xlibrary/assets/ small-vessel-security-strategy.pdf.

[2] M. Bruno, K. W. Chung, H. Graber et al., "Concurrent use of satellite imaging and passive acoustics for Maritime domain awareness," in Proceedings of the Waterside Security Conference, Marina di Carrara, Italy, November 2010.

[3] V. Bush, J. B. Conant, and J. T. Tate, "Principles and applications of underwater sound," Tech. Rep., Office of Scientific Research and Development, Washington, DC, USA, 1946, Vol 7. Summary, http://handle.dtic.mil/100.2/AD200786.

[4] E. Miasnikov, "What is Known About the Character of Noise Created by Submarines?" Publihed in The Future of Russia's Strategic Nuclear Forces: Discussions and Arguments, 1998, http://www.armscontrol.ru/subs/snf/snf03221.htm. 
[5] Underwater Radiated Noise of Ocean-Going Merchant Ships, "A Background Paper Produced by Participants of the International Workshop on Shipping Noise and Marine Mammals," Held By Okeanos: Foundation for the Sea. Hamburg, Germany, April 2008, http://www.okeanos-stiftung.org/assets/ Uploads/BiologyPaper2.pdf.

[6] D. Ross, Mechanics of Underwater Noise, Pergamon, New York, NY, USA, 1976.

[7] J. S. Carlton and D. Vlasic, "Ship vibration and noise: some topical aspects," in Proceedings of the 1st International Ship Noise and Vibration Conference, London, UK, June 2005, Lloyd's Register Technical Papers.

[8] S. J. Malinowski and I. Gloza, "Underwater noise characteristics of small ships," Acta Acustica United with Acustica, vol. 88, no. 5, pp. 718-721, 2002.

[9] A. Zak, "Ships classification basing on acoustic signatures," WSEAS Transactions on Signal Processing, vol. 4, no. 4, pp. 137149, 2008.

[10] R. K. Lennartsson, E. Dalberg, D. Lindgren, and L. Persson, "Improved classification ability in littoral environments by decision fusion," in Proceedings of the OCEANS-Europe Conference, June 2007, article 4302298.

[11] F. B. Tuteur, "Detection of wide-band signals modulated by a low-frequency sinusoid," in Processing of Data from Sonar Systems, R. A. MacDonald et al., Ed., Appendix A-4, Yale University, New Haven, Conn, USA, 1963, AD-420575.

[12] H. A. D'Assupcao, "Theoretical assessment of DEMON performance," Technical Memorandum WRE-CPD-TM-169, 1970, http://dspace.dsto.defence.gov.au/dspace/handle/1947/ 9119.

[13] A. A. Kudryavtsev, K. P. Luginets, and A. I. Mashoshin, "Amplitude modulation of underwater noise produced by seagoing vessels," Acoustical Physics, vol. 49, no. 2, pp. 184$188,2003$.

[14] A. Kummert, "Fuzzy technology implemented in sonar systems," IEEE Journal of Oceanic Engineering, vol. 18, no. 4, pp. 483-490, 1993.

[15] F. Bao, X. Wang, Z. Tao, Q. Wang, and S. Du, "Adaptive extraction of modulation for cavitation noise," Journal of the Acoustical Society of America, vol. 126, no. 6, pp. 3106-3113, 2009.

[16] R. Rajagopal, B. Sankaranarayanan, and P. Ramakrishna Rao, "Target classification in a passive sonar-an expert system approach," in Proceedings of the International Conference on Acoustics, Speech, and Signal Processing: Speech Processing, vol. 5, pp. 2911-2914, April 1990.

[17] S. Li and D. Yang, "DEMON feature extraction of acoustic vector signal based on 3/2-D spectrum," in Proceedings of the 2nd IEEE Conference on Industrial Electronics and Applications (ICIEA '07), pp. 2239-2243, May 2007.

[18] L. Fillinger, A. Sutin, and A. Sedunov, "Cross-correlation of ship noise for water traffic monitoring," Tech. Rep., Acoustical Society of America, 158th Meeting Lay Language Papers, 2009, http://www.acoustics.org/press/158th/fillinger.htm.

[19] L. Fillinger, A. Sutin, and A. Sedunov, "Acoustic ship signature measurements by cross-correlation method," Journal of the Acoustical Society of America, vol. 129, no. 2, pp. 774-778, 2011.

[20] R. Stolkin, A. Sutin, S. Radhakrishnan et al., "Feature based passive acoustic detection of underwater threats," in Photonics for Port and Harbor Security II, vol. 6204 of Proceedings of SPIE, pp. 40-49, April 2006.

[21] A. Sutin and B. Bunin, "Acoustic research for port protection at the Stevens Maritime Security Laboratory," in Proceedings of the 3rd International Conference and Exhibition on Underwater Acoustic Measurements: Technologies \& Results, Nafplion, Greece, June 2009.

[22] A. Sutin, B. Bunin, A. Sedunov, N. Sedunov, M. Tsionskiy, and M. Bruno, "Stevens passive acoustic system for underwater surveillance," in Proceedings of the Waterside Security Conference, Marina di Carrara, Italy, November 2010.

[23] Sevens Summer Reaserch Institute (SRI), Stevens Institute of Technology, 2010, http://www.stevens.edu/csr/education/ Summer_Research_Institute.html. 

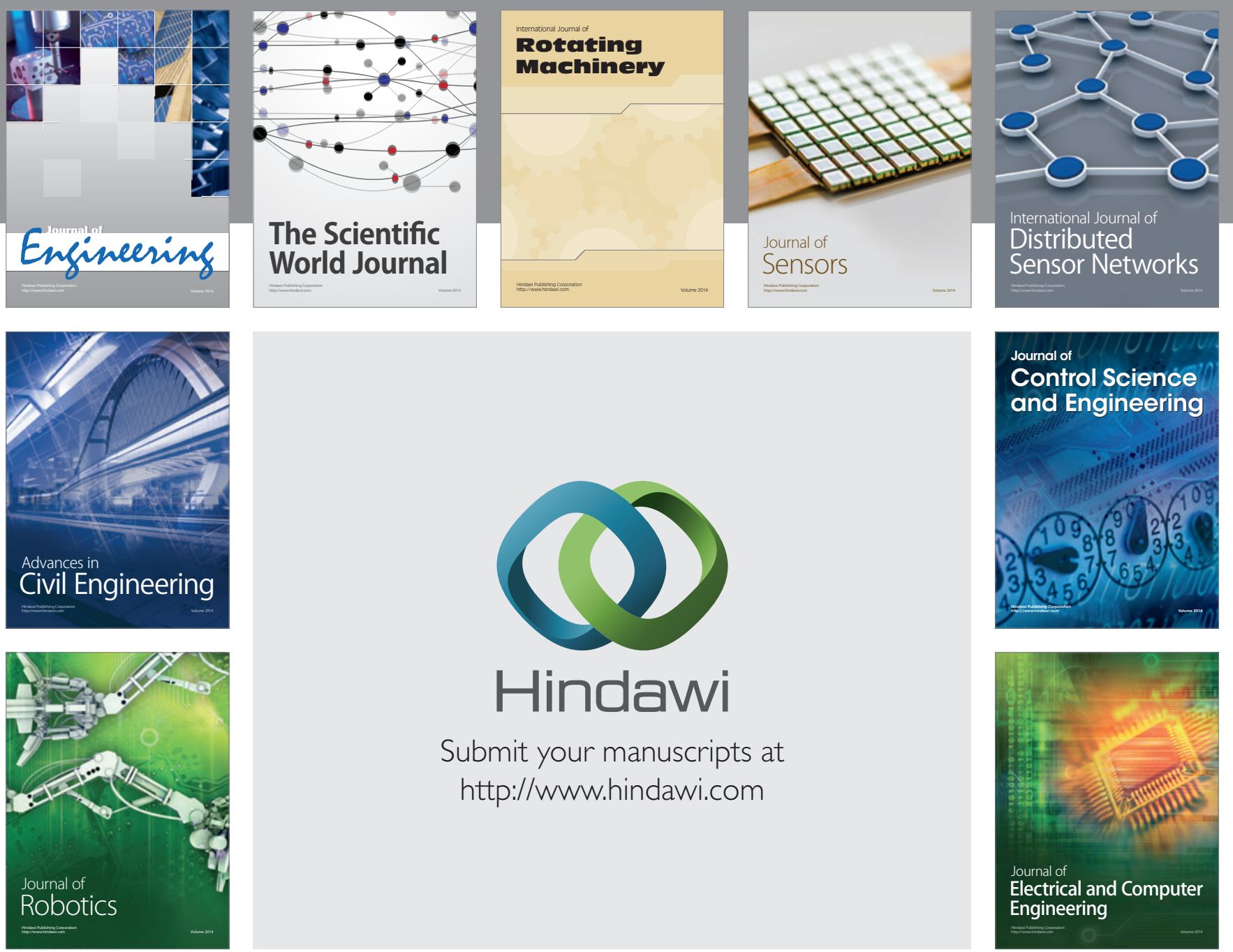

Submit your manuscripts at

http://www.hindawi.com
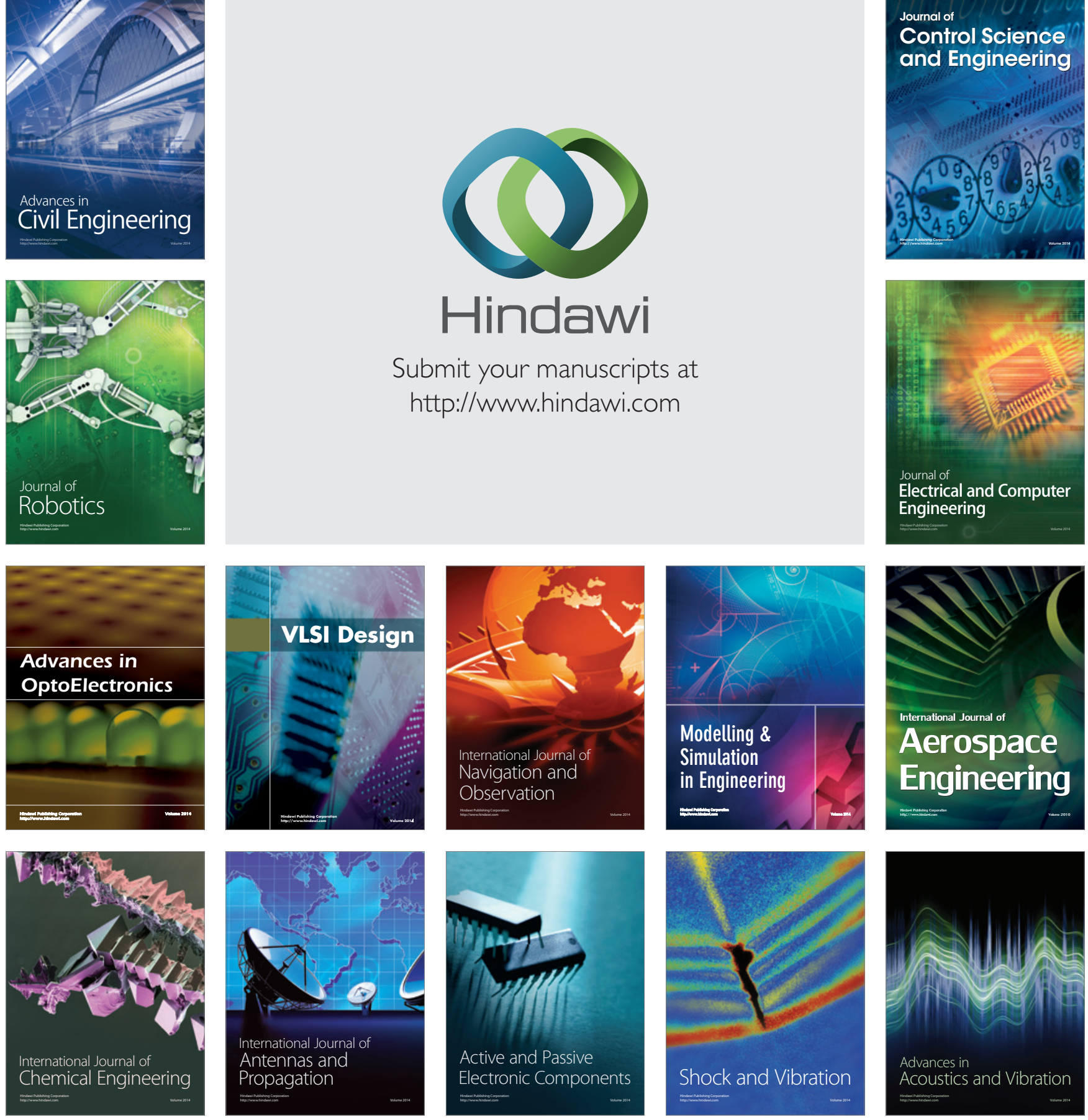